\title{
Anomalies in Geomagnetic Variations in the Arctic Archipelago of Canada
}

\author{
By K. WHITHAM \\ Dominion Observatory, Ottawa, Canada
}

\begin{abstract}
Two unusual features of geomagnetic variations have now been discovered in the Arctic Archipelago of Canada. The first anomaly at Alert on Ellesmere Island is characterized by abnormally high levels of irregular magnetic activity and a persistent directional characteristic of the vector horizontal disturbance. An adequate ionospheric explanation has not been found. Recent papers have given an explanation of the gross characteristics of the Alert magnetograms in terms of induction in a large anomalous conductor in the upper mantle striking parallel to the channel separating north Ellesmere Island from Greenland. Field experiments followed by simple potential and spectral response analyses confirm the existence of a gross conductor. In this paper the uncertainties in and shortcomings of the first-order explanation and minor modifications are stressed, and an alternative solution involving induction in the conducting mantle and a highly conducting circuit examined. The alternative solution appears to be even more inadequate.

The second anomaly at Mould Bay, Prince Patrick Island, is characterized by the striking absence of shorter-period vertical field magnetic variations. Assuming the presence of a sheet conductor at depth it has been estimated earlier from the frequency dependence of the power spectrum that a $10-20 \mathrm{~km}$ thick layer with a conductivity near $10^{-11} \mathrm{emu}$ is required near the bottom of the crust or in the upper mantle. The assumptions in deriving this explanation are outlined, and theoretical curves shown which demonstrate that the neglect of reasonable conductivity above and below the anomalous layer appears justified. Results are shown applying the same technique to plane earth induction in a non-anomalous area, and to one magneto-telluric situation of current interest.
\end{abstract}

\section{Introduction}

Unusual features of geomagnetic variations have now been noted at two locations in the Arctic Archipelago of Canada, and attributed to anomalous induction within the earth. At Alert on Ellesmere Island, the anomaly is characterized by abnormally high levels of irregular magnetic activity and a pronounced directional characteristic of the vector horizontal disturbance. Whitham and Andersen (1962) concluded that the effects could not be satisfactorily explained in terms of ionospheric sources, and that the magnitude, directional characteristics and frequency dependence of the anomaly were in general agreement with the hypothesis of an anomalous highly conducting body striking $N E-S W$ with its centre 
to the $S E$ of Alert. Law et al. (1963) have described simple field experiments designed specifically to test this hypothesis in a logistically difficult region. The field results from stations along a line perpendicular to the strike were subjected to simple potential analysis, and showed that the anomalous internal contributions to the magnetic variation vector were approximately consistent with an underground current in a $N E-S W$ direction at a depth between 50 and $70 \mathrm{~km}$, a few $\mathrm{km} S E$ of Alert. Approximating the anomalous conductor by a cylinder of uniform conductivity and infinite length, they showed that the horizontal field response as a function of frequency could be explained with a conductivity of $3 \times 10^{-11}$ $\mathrm{emu}$ and a cylindrical radius of about $50 \mathrm{~km}$. The gravitational consequences of such a model were examined under the assumption of hydrostatic equilibrium, and crude agreement obtained with a Bouguer anomaly found in the same region, assuming certain reasonable regional gradients. Law et al. (1963) mentioned an asymmetry found experimentally and suggested a qualitative explanation for this. In this paper the techniques used in making a scientific interpretation of the Alert anomaly are reviewed and the uncertainties in the conclusions of Law et al. (1963) clearly stated. Preliminary results of a more general discussion of induction in large bodies are outlined, and short-comings in the present solution clarified. An alternative explanation in terms of induction in the mantle and the presence of a highly conducting circuit is attempted but this appears to be even more inadequate as an explanation. The need for better and more extensive experimental data is clear.

The second unusual feature was noted at Mould Bay, Prince Patrick Island where standard-run magnetograms demonstrate a striking absence of vertical field magnetic variations. Whitham (1963) has calculated from his estimate of the anomalous steepening of the vertical field power spectrum that the effect could be produced by a $20 \mathrm{~km}$ thick layer in the crust with a conductivity near $10^{-11} \mathrm{emu}$. The interpretation must be regarded as speculative. Since no evidence for a highly conducting crust exists, anomalous temperatures difficult to reconcile with a stable region are required to explain the anomaly by semi-conduction processes, and the interpretation made depends upon a number of unproved, if reasonable, assumptions.

In this paper these assumptions are outlined, and one assumption is tested by extending the calculations to estimate the frequency dependence of the power spectrum for a multiple layered earth. The results of extensive calculations suggest that the parameters estimated by the simpler method, mentioned above, may require modification by factors of two only in thickness and that it is difficult to obtain theoretical agreement (under present assumptions) with a conductor at any great depth.

The technique used in making the second approximation to the situation at Mould Bay can be applied to obtain reasonable results in a non-anomalous area and to consideration of magneto-telluric models. Some preliminary results of current interest will be given.

\section{Polar Cap Magnetic Variation Anomalies}

Scientific investigation of the features attributed to unusual induction inside the earth and described above presents novel problems at these high latitudes, where climate, ease of access and morphological characteristics of magnetic disturbance are very different from 
those at locations where other anomalies have been defined. However, interest is just as great in the Arctic in quantitative interpretation, delineating the extent and assessing the tectonic significance, if any. Consequently it appears useful to explain the basic procedures used in much of our work: basic differences will be noted from the approaches of Rikitake (1959a) and Schmucker (1959) to the two best defined anomalies.

The dominant feature of all polar cap magnetograms is the existence of wide-band irregular vector magnetic disturbance having a primary broad day-time maximum in the Arctic summer. Clear events such as crochets, or bays with sharp commencements are comparatively rare or non-existent, superposed disturbance indicating great source complexity appears to be usual, and the density of permanent magnetic observatories or variation stations is light (1 per $10^{6} \mathrm{~km}^{2}$ in the Canadian and Danish sectors north of geomagnetic latitude $79^{\circ} \mathrm{N}$ ). Since the auroral zone with its complex and different pattern of disturbance lies to the south, it appears to be impossible to attempt the usual kind of "event" potential analyses to define magnetic anomalies. Whitham and Andersen (1962) have summarised the difficulties. Furthermore logistic difficulties and the great expense of Arctic operations prevent the operation of a network of variometers over long periods of time.

Consequently our approach to the problems has been weighted to the use of the record as a whole rather than by event analysis and extensive potential analysis. Range measurements, spectral analyses and analogies with electromagnetic prospecting techniques have proved useful tools. The assumptions made in this approach can usually be specifically defined, and on occasion are different from the more usual ones. For example in the polar cap it appears quite unfounded to assume the customary middle-latitude approximation that the normal induced vertical field variation cancels the inducing vertical field variation for frequencies $10^{-2}$ to $10^{-4}$ c.p.s. A further reason for the approach adopted is that equipment drift and temperature effects coupled with logistic limitations have severely limited the available length of records at field sites and made the interpretation at diurnal and semidiurnal periods uncertain. Techniques are however being developed to overcome all these problems.

\section{The Alert Anomaly in Geomagnetic Variations}

The results of the field work described by Law et al. (1963) can be summarized as follows:

1) The $\mathrm{N} 45^{\circ} \mathrm{E}$ strike of the anomalous body at Alert is confirmed, and a large anomaly exists in the amplitude of the variations in the horizontal field component perpendicular to strike $\left(\Delta H_{1}\right)$. Visual examination of magnetograms suggested that the half-width of the anomaly is some 50 to $100 \mathrm{~km}$. Range measurements and examinations of the records show that whereas the $\Delta H_{1}$ effect is very approximately symmetrical about Alert along a line perpendicular to strike, the $\Delta Z$ effect is asymmetric, the vertical field fluctuations on the $S E$ (Greenland) side of Alert exceeding those on the $N W$.

2) The simple two dimensional potential analysis clearly demonstrates the existence of an anomalous internal component. Although many approximations are involved, particularly with a minimum number of stations, the analysis is consistent with a current 
flowing $N W-S E$ at a depth between 50 and $70 \mathrm{~km}$, a few $\mathrm{km} S E$ of Alert.

3) If an independent assumption is made that the effect can be approximated by electromagnetic induction within an isolated uniform infinite cylinder, the horizontal field response as a function of frequency appears to be consistent with a conductivity $\sim 3 \times 10^{-11} \mathrm{emu}$ and radius $\sim 50 \mathrm{~km}$. It was also noted that, in the sample calculated, the vertical field variation short-period ( $<10$ to 15 minutes) response at Alert was too great for this particular model.

4) The neglect of the conducting mantle was noted and it was estimated that a rise of about $100 \mathrm{~km}$ over a lateral distance of about $300 \mathrm{~km}$ in the isotherm corresponding to $10^{-11}$ emu conductivity $\left(1400^{\circ} \mathrm{C}\right)$ could account for the anomaly. The asymmetries mentioned above were believed to correspond to the effect of a steeper slope of the isotherms plunging $S E$ at Alert.

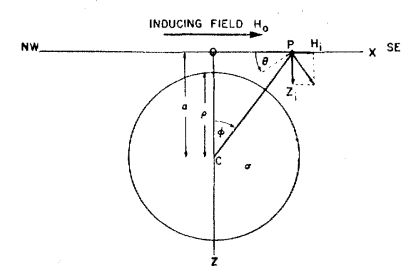

Fig. 1. Illustrating the geometry for an isolated cylinder, radius $\rho$, conductivity $\sigma$ and unit permeability striking $N E$ -

$S W$ with centre at depth $a$.

Additional computations have since been made. With the notation of Figure 1, for periods for which the conductivity of the cylindrical body is effectively infinite and for a uniform horizontal inducing field $H_{0} e^{j \omega t}$ perpendicular to strike, it can easily be seen from the formulae of Whitham and Andersen (1962) that at point $P, H_{i} / Z_{i}=$ $\operatorname{cotan} 2 \phi$. Therefore $\theta=\pi / 2-2 \phi$. The maximum horizontal field response occurs directly above the centre of the body, $H_{i} \max / H_{0}=(\rho / a)^{2}$ and the maximum vertical field response $Z_{i} \max / H_{0}=3 \sqrt{3}(\rho / a)^{2} / 8$ occurs at a horizontal distance $\pm a / \sqrt{3}$ from the centre, where $H_{i} / H_{0}=3 / 8(\rho / a)^{2}$. These considerations suggest that the probable results of potential triangulation of short period events is the detection of the top of the anomalous body, for the station separations used by Law et al. (1963). Calculations of Rikitake (1963) for other two dimensional bodies of different crosssection suggests this result is generally true as a first approximation.

However a clear difficulty emerges at Alert if the top of the responsible conductor is placed at or near a depth of $60 \mathrm{~km}$. Spectral calculations and earlier range estimates show that $(\rho / a) \sim 0.85$ is required to explain the magnitude of the anomaly. Therefore if $0.15 a$ is equated to $60 \mathrm{~km}, a \sim 400 \mathrm{~km}$ and $\rho \sim 340 \mathrm{~km}$ and the experimentally determined halfwidth of the anomaly is much too small. For these reasons Law et al. (1963) considered induction in a body of $\sim 50 \mathrm{~km}$ cylindrical radius with its top within $10 \mathrm{~km}$ of the surface. The experimental half width is then explained, but the triangulation depth of $60 \mathrm{~km}$ must be regarded as largely fortuitous.

Figure 2 shows schematically an experimentally determined estimate of the horizontal and vertical field response along a NW-SE section through Alert. The estimates were made using the half-hourly and hourly range measurements of all available records and the potential triangulation results. It is believed that the horizontal field response inferred is unlikely to be in error by more than 0.2 ; the vertical field response shown is likely to be in error by as much as 0.2 , since depending on the assumptions made, the vertical field response 


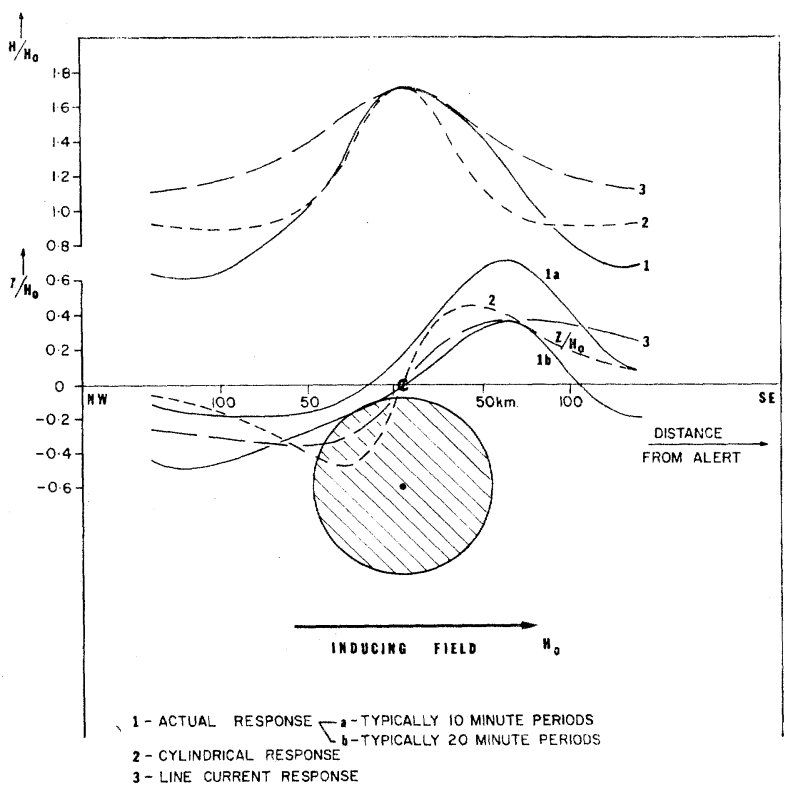

Fig. 2 Estimated approximate horizontal and vertical field response along a $N W-S E$ section, together with the normalized theoretical response of a line current at $60 \mathrm{~km}$ depth and the cylindrical model of Law et al. (1963). The increasing asymmetry of the short period vertical field response is indicated.

can be peaked higher to the $S E$. This is shown in the figure where the estimated response is shown schematically at 10 and at 20 minute periods. The theoretical response of a line current normalized to fit the horizontal field at Alert is shown together with that of the first model of Law et al. (1963). The experimental data is quite inadequate to distinguish between the two cases.

Rikitake's calculations (1963) for an elliptical section and a thin wedge suggest that the decay of the vertical field response can be made asymmetric in the required sense without seriously distorting the horizontal field symmetry: since sufficiently precise quantitative description of the observed effect is not yet possible with the records available, different models all of which are consistent with the isotherms plunging more gradually to the $N W$ cannot be distinguished. The half-width of the horizontal field response is 0.97 times the depth to centre for a cylinder: Rikitake's specific elliptical and wedge models exhibit halfwidths $\sim 0.8$ to 0.9 times the depth to centre. It seems clear that the experimental data of Law et al. (1963) could be made consistent with induction in circular or elliptical cylinders or wedge shaped bodies with centres as deep as $100 \mathrm{~km}$ (or a rise of up to $200 \mathrm{~km}$ in the $1400^{\circ} \mathrm{C}$ isotherm), and the cross-sectional shape is not of great significance. However the magnitude of the effect requires a very high conductivity very close to the surface.

The latter requirement implies very high temperatures abnormally close to the surface and these are difficult to reconcile with a stable region. Again the length of the anomalous conductor along strike is presently unknown although under experimental examination. 
In the treatments above it has been tacitly assumed that the strike length is comparable to or exceeds typical scale lengths of the inducing field. Such enormous lengths (500 to $1000 \mathrm{~km}$ ) are comparable to the length of the Sverdrup basin, or to the separation of mag. netic observatories in the polar cap. Consequently it appears worthwhile examining the alternative idea of induction inside the conducting mantle driving currents through a conducting circuit striking $S W \cdot N E$ at a depth of about $60 \mathrm{~km}$. Rikitake (1959 b) has studied the branch circuit problem under the gross approximation that induction in the circuit itself can be neglected. Suppose a cylindrical conductor of radius $\rho_{c}$, conductivity $\sigma_{c}$ is connected to two points in the mantle separated by $l$ in the $S W$-NE direction. The centre of the conductor is at a depth $a$ below the surface. For a uniform inducing field $H_{0} e^{\text {iwt }}$ in the direction $\theta=0$, the azimuthal electric field induced at the boundary of the conducting sphere of radius $R$, conductivity $\sigma$ can be written, following Rikitake (1959b)

$$
E / i \omega H_{0}=-F_{1}(k R) R \sin \theta / 2 F_{0}(k R)
$$

with

$$
k^{2}=4 \pi i \sigma \omega, F_{0}(x)=\sinh x / x, F_{1}(x)=3(\cosh x-\sinh x / x) / x^{2}
$$

Taking

$$
R=6 \times 10^{3} \mathrm{~km}, \sigma=2 \times 10^{-12},|k R|>1 \text { for } T \ll 100 \mathrm{hrs}
$$

and

$$
F_{1}(k R) / F_{0}(k R) \approx 3 / k R
$$

Therefore $E / H_{0}=-3 \sqrt{2} e^{i \pi / 4} / 4(\sigma T)^{1 / 2}$ for a uniform horizontal inducing field.

Therefore

$$
\left|\frac{H_{i}}{H_{0}}\right|=\frac{2 i}{a H_{0}}=\frac{2}{a} \frac{E l}{H_{0}} \frac{\sigma_{c} \pi \rho_{c}^{2}}{l}=\frac{\pi \rho_{c}^{2} \sigma_{c}^{3} \sqrt{2}}{2 a(\sigma T)^{1 / 2}}
$$

Since at Alert

$$
\frac{H_{i}}{H_{0}} \sim 0.7 \text { at } T \sim 10^{3} \text { secs, }
$$

if $\sigma=2 \times 10^{-12}, \rho_{c} \sigma_{c}{ }^{1 / 2} \sim 5$ and if $\sigma=10^{-11}, \rho_{c} \sigma_{c}{ }^{1 / 2} \sim 11$. For a conducting circuit with $\sigma_{c} \sim$ $10^{-10} \mathrm{em} \mathrm{u}$, a diameter of 10 to $22 \mathrm{~km}$ is required. The calculations of Law et al. (1963) indicate a size-conductivity parameter of 27 . It is obvious therefore that induction in the requisite branch circuit itself cannot be neglected.

The above treatment for a uniform inducing field can be modified to take account of the finite scale length of the real inducing field following the method of Price (1962). Consider a plane earth approximation with a conducting mantle, conductivity $\sigma$, at depth $h$ below the surface $z=0$. For an elementary source potential of form $\emptyset=A e^{-\nu z} P(x, y, \nu)$, neglecting the displacement current, the vector magnetic field $\underline{H}$ satisfies the equation

$$
i \omega \underline{H}=-e^{i \omega t}\left(\frac{d W}{d z} \frac{\partial P}{\partial x}, \frac{d W}{d z} \frac{\partial P}{\partial y}, \nu^{2} W P\right)
$$

where $W$ satisfies the equation $\frac{d^{2} W}{d z^{2}}=\left(\nu^{2}+4 \pi i \omega \sigma\right) W$ inside the mantle and $\frac{d^{2} W}{d z^{2}}=\nu^{2} W$ outside the conductor, and $P$ satisfies the equation $\frac{\partial^{2} P}{\partial x^{2}}+\frac{\partial^{2} P}{\partial y^{2}}+\nu^{2} P=0$ where $\nu$ is a constant (equal to $2 \pi$ /wavelength of field).

Inside the conductor $\underline{E}=e^{i \omega t} W(z)\left(\frac{\partial P}{\partial y}, \frac{-\partial P}{\partial x}, 0\right)$ 
Defining $\theta^{2}=\nu^{2}+4 \pi i \omega \sigma, W=A e^{\nu z}+B e^{-\nu z}$ outside the conductor and $W=C e^{-\theta z}$ inside the mantle.

The boundary conditions at $z=h$ lead to

$$
\begin{gathered}
A e^{\nu h}+B e^{-\nu h}=C e^{-\theta h} \\
\nu\left(A e^{\nu h}-B e^{-\nu h}\right)=-\theta C e^{-\theta h}
\end{gathered}
$$

and

$$
E_{x}(z=h) / i \omega H_{y}(z=0)=\frac{-C e^{-\theta h}}{\nu(A-B)}=\frac{2}{(\theta+\nu)\left(e^{\nu h}+e^{-\nu h}\right)-2 \nu e^{-\nu h}}
$$

For

$$
\sigma>2 \times 10^{-12}, T \ll 240 \mathrm{~m}, \nu<10^{-7} \mathrm{cms}^{-1}
$$

$$
\theta \approx(1+\nu) 2 \pi(\sigma / T)^{1 / 2}
$$

and

$$
\begin{gathered}
E_{x}(z=h) / H_{y}(z=0)=2 i \omega e^{-\nu h} /(1+i) 2 \pi(\sigma / T)^{1 / 2} \quad \text { when } 2 \nu h \gg 1 \\
=(1+i) e^{-\nu h} /(\sigma T)^{1 / 2}=\sqrt{2} e^{i \pi / 4} e^{-\nu h} /(\sigma T)^{1 / 2}
\end{gathered}
$$

which differs by a factor $\alpha=\frac{3}{4} e^{-v h}$ from the result obtained on the uniform-field excitation approximation. For $\nu=10^{-7} \mathrm{~cm}^{-1}, h=600 \mathrm{~km} \alpha=10^{-3}$ : taking the most optimistic estimate $\nu=5 \times 10^{-8} \mathrm{~cm}^{-1}, h=200 \mathrm{~km}, \alpha \sim 0.3$. The mechanism seems to be inadequate to explain the observed effect unless the conducting part of the mantle rises enormously, and even then too localised fields are incapable of producing effects larger than those produced by induction in the body itself.

Other difficulties with the branch-circuit hypothesis arise from the predicted phase shift of $45^{\circ}$, since at shorter periods the response appears to be more in-phase than phaseshifted, and from the predicted frequency dependence. In the range where the approximations outlined in the above treatment should be valid, the theoretical response should vary with $\omega^{\frac{1}{2}}$. Figure 6 of Law et al. (1963) is not in very good agreement with this hypothesis, although it now appears clear that a more precise determination of the frequency dependence may lead to the clearest way of distinguishing between the two general hypotheses.

\section{The Mould Bay Anomaly in Geomagnetic Variations}

Whitham (1963) has derived by spectral analysis a numerical description of the unusual frequency-dependent attenuation of the vertical field component at Mould Bay, and shown by general arguments from the theory of Price (1962) that an anomalous conductor in the crust or upper mantle with thickness $20 \mathrm{~km}$ and conductivity $10^{-11} \mathrm{e} \mathrm{mu}$ is necessary to explain the results. The method used to estimate the conductor depends on a number of assumptions: (1) The anomalous body is assumed to be a plane sheet effectively of infinite extent in terms of the typical scale lengths of the inducing field. The only justification to date for this assumption is that no structure can be inferred from the Mould Bay magnetograms, in contrast to the development at Alert, and that experimental work to determine the extent is only now underway. (2) The frequency attenuation curve derived is assumed to be substantially time-invariant, and therefore attributable to induction inside the earth. The empirical arguments for the validity of this assumption have been given by 
Whitham (1963); it is probable that much more extensive machine computing would be required to improve substantially the curve. (3) A time-independent mean field wavelength is assumed in fitting theoretical curves to the observed frequency attenuation of the vertical field fluctuations. At the present time this assumption cannot be justified except as a reasonable approximation in view of the statistical nature of the analysis, and a requirement if further progress is to be made using the technique outlined by Whitham (1963). (4) The effect of non-anomalous conductivity both above and below the anomalous body is neglected.

This latter assumption has now been examined by further calculations using a digital computer. Assuming an elementary source field with potential $\Phi=A e^{-\nu z} P(x, y, \nu)$ where $P$ satisfies the equation $\frac{\partial^{2} P}{\partial x^{2}}+\frac{\partial^{2} P}{\partial y^{2}}+\nu^{2} P=0$, that part of the theoretical power density ratio (vertical to horizontal field fluctuation) independent of $P$ has been calculated for a number of models, and its frequency variation compared with the estimate made from the power spectral content of typical magnetograms from Mould Bay.

Figure 3, the legend of which is self-explanatory, show the results of five models out of more than one hundred now examined. Models 010,020 represent the earlier solution of Whitham (1963). Models 030,040 represent at least as adequate a fit and Model 050 illustrates that a change of 2 in the mean wavelength estimate does not significantly change the estimate of a $10-20 \mathrm{~km}$ thick conductor, conductivity $10^{-11} \mathrm{em} \mathrm{u}$, in the crust or upper

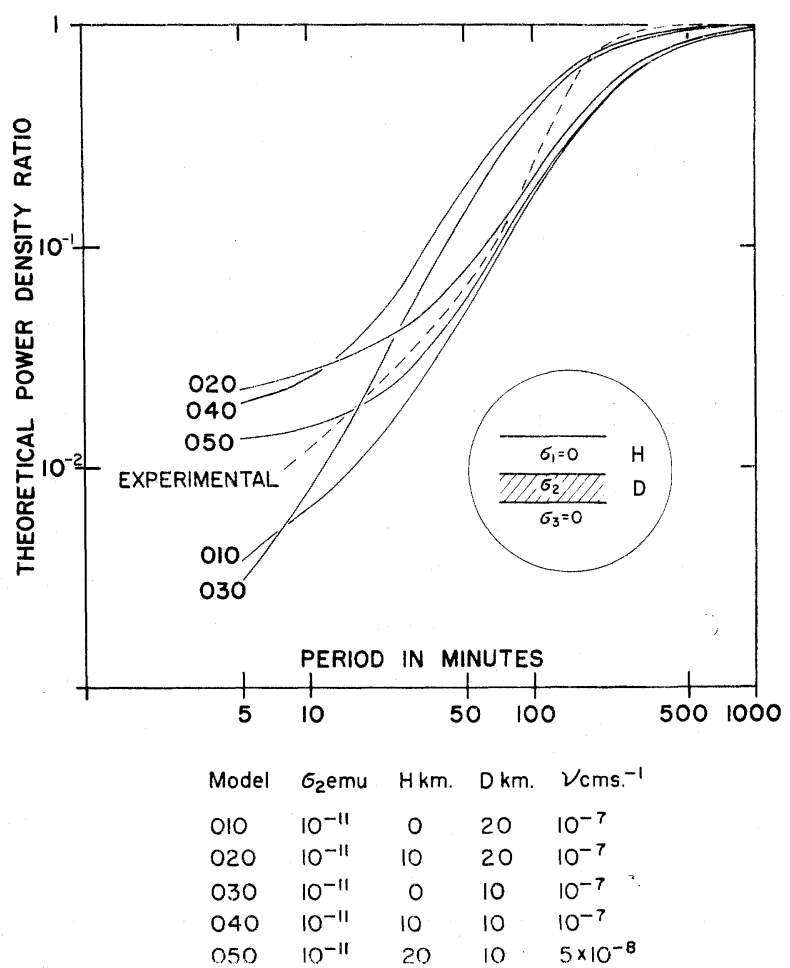

Fig. 3 Calculated power spectral ratios for typical isolated conductor cases. 
mantle. In the different models calculated, the thickness of the conductor has varied from 2 to $20 \mathrm{~km}$, the conductivity from $10^{-10}$ to $10^{-11} \mathrm{e} \mathrm{mu}$ and the depth to the top of the conductor from 0 to $100 \mathrm{~km}$.

The observed flattening of the response at long periods, and the probable reduction in slope at shorter periods limits the range of acceptable models to those shown for single layer calculations. Because of the difficulty in accounting for such high values of conductivity at such shallow depths (as mentioned for Alert also), particular attention has been paid to the theoretical effect of extensive near-surface salt beds. Provided the wavelength of the inducing field is sufficiently small an approximate fit at longer periods can be obtained: such a fit appears incompatible with the observed decrease in slope at shorter periods.

Models were next examined with realistic estimates of conductivity above and below the conducting body. Figure 4 shows the results of assuming a conductivity $10^{-14}$ to $10^{-12}$ e m u for the $20 \mathrm{~km}$ layer above Model 050 . The effect of surface values $10^{-14}$ to $10^{-13} \mathrm{e} \mathrm{mu}$ is seen to be unimportant: the introduction of vast thicknesses of sediment with conductivity $10^{-12}$ e mu produces changes of less than $50 \%$ at 10 minute periods. It is concluded that the neglect in the earlier work of the surface section of a few $\mathrm{km}$ of sediment underlain by a crystalline basement was quite justified.

Figure 5 shows the effect of finite conductivity below two of the reasonable models mentioned above. Models 020,050 modified by a near surface conductivity value of $10^{-14}$

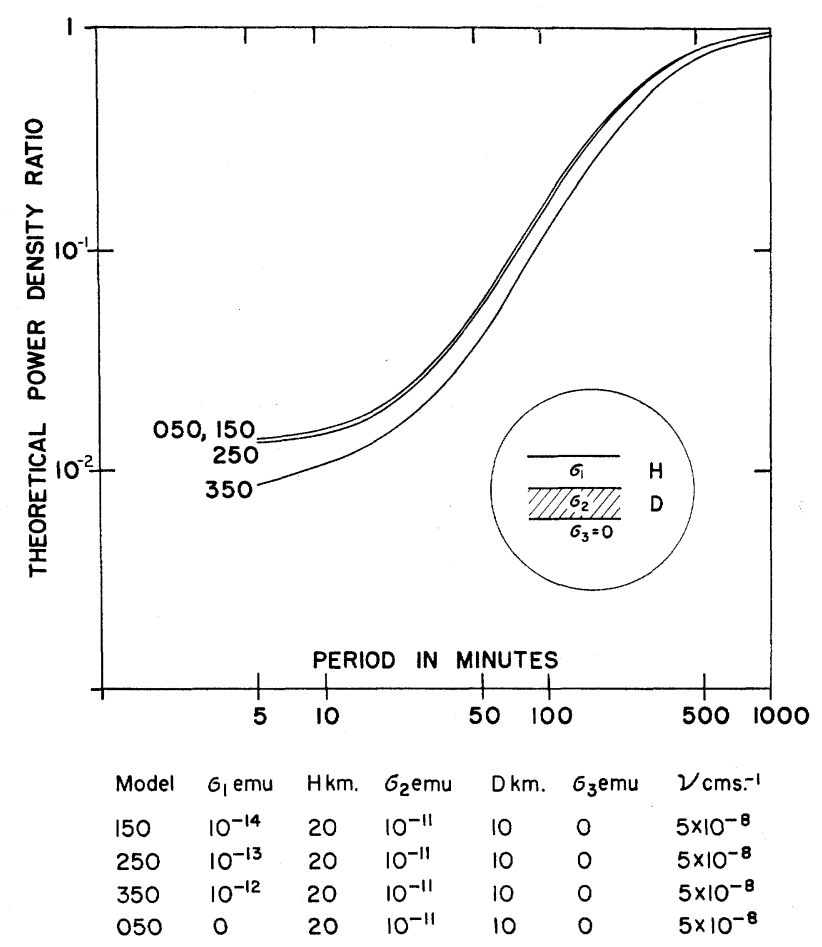

Fig. 4 Calculated power spectral ratios for a typical conductor introducing finite conductivity into the surface layers. 


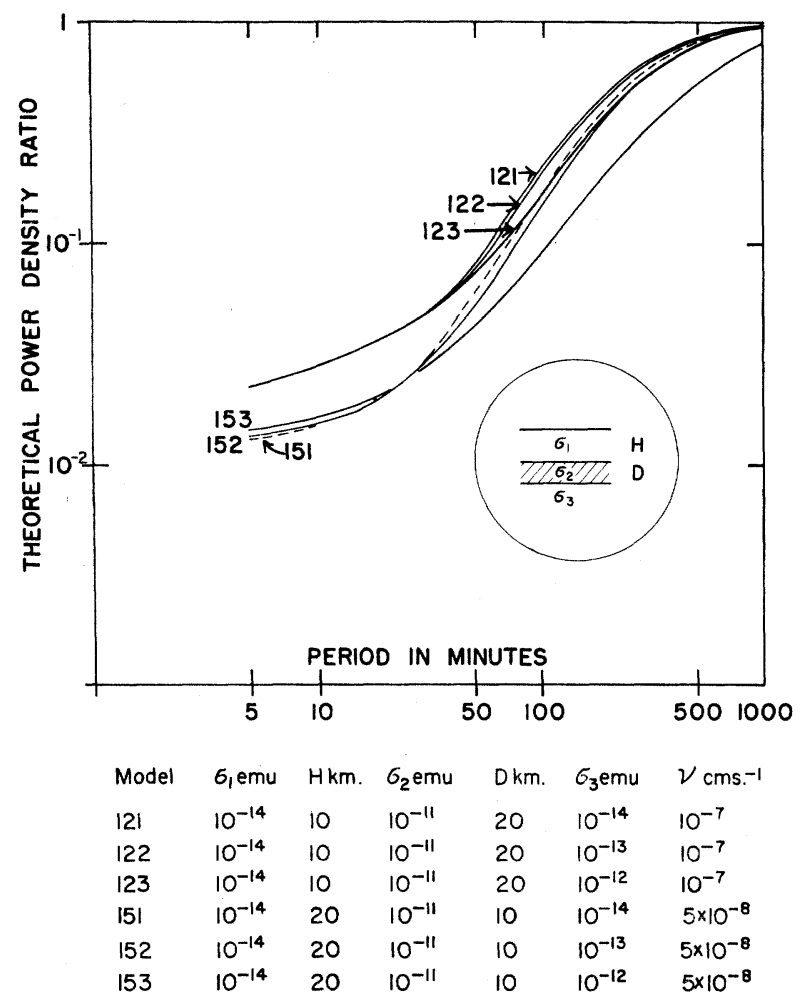

Fig. 5 Calculated power spectral ratios for two typical conductors introducing finite conductivity above and below the body.

$\mathrm{e} \mathrm{mu}$ and third layer values of $10^{-14}$ to $10^{-12} \mathrm{e} \mathrm{mu}$ are shown. The legend is self-explanatory. Model 151 is indistinguishable from 050 and 121 from 020. The influence of a relatively high conductivity, such as $10^{-13} \mathrm{e} \mathrm{mu}$, below the body is unimportant, but a value of $10^{-12}$ e $\mathrm{m} u$ has a marked effect for periods longer than about 30 minutes. Unfortunately, as would be predicted, the effect increases the discrepancy at longer periods between the experimental results and the best-fitting models. The conclusion is clear: the fourth assumption outlined earlier was essentially justified. Until experimental investigations of the extent of the Mould Bay anomaly are complete, further extensive calculations are probably not worthwhile.

However, it does appear worthwhile to consider the relevance of the calculations now made to magnetotelluric studies in western Canada, and to induction in a non-anomalous area. Price (1962) in his discussion of magnetotelluric methods when the source field is considered has presented the relevant equations. At the surface of the earth $E_{x} / i \omega H_{y}$ $=-\left\{\frac{W(z)}{d W}\right\}_{z=0,}$ whereas for three-layer cases the author has computed $\left.\left\{\frac{\frac{\nu W}{d z}}{d z}\right\}_{z=0}\right|^{2}$ as a function of $\sigma_{1}, H, \sigma_{2}, D, \sigma_{3}, \nu$ and $T$ in the pass-band from 10 to $10^{3}$ minutes. The theoretical curves of the writer can thus be converted to plots of apparent resistivity versus period, such as are usually displayed in magnetotelluric sounding, by multiplication by 
$\omega^{2} 2 T / \nu^{2}=8 \pi^{2} / T \nu^{2}$. There has recently been considerable discussion of some magnetotelluric results which indicate a rise in conductivity at comparatively shallow depths of 70 to $200 \mathrm{~km}$. For a location in western Canada such as Meanook, models of interest consist of $2 \mathrm{~km}$ of sediment, conductivity $2 \times 10^{-12} \mathrm{e} \mathrm{m} \mathrm{u}$, underlain by an unknown thickness with conductivity as low as that typical of crystalline basement rocks, perhaps $10^{-14} \mathrm{emu}$. The 'ohmo' or level where the conductivity is believed to increase is often estimated on the fundamental assumption that $\nu=0$ and is variously placed at depths from 70 to $200 \mathrm{~km}$ with a conductivity contrast of 5 to 10. Figure 6 shows the theoretical curves for a number of models $M 1$ to $M 8$ typical of such a structure. In different models, wavelengths from 600 to $6000 \mathrm{~km}$ are adopted - the legend is self-explanatory. For four cases, the apparent resistivity deduced as described above is shown. The theoretical curves show immediately for all wavelengths within this range the difficulties in resolving between a 90 and $200 \mathrm{~km}$ depth. Furthermore the apparent resistivity curves indicate that the nature of the decrease in apparent resistivity at periods of several minutes (often the basis for estimating a shallow ohmo) is heavily dependent on the wavelength of the field. It is clear that magnetotelluric solutions made under the assumption $\nu=0$ should be examined to see if the results are compatible with the $H z / H x$ ratios at the station. For example a cursory inspection of Meanook

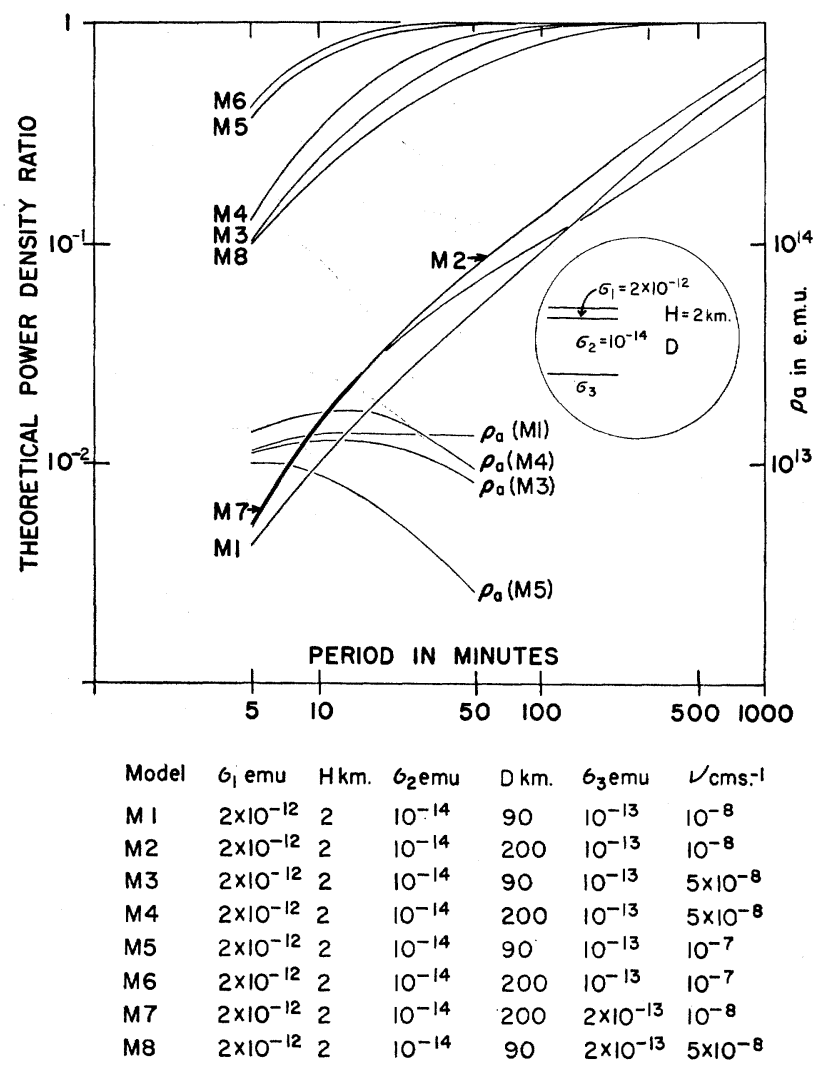

Fig. 6 Calculated power spectral ratios and apparent resistivity for a theoretical section typical of one in Western Canada derived from magnetotelluric analysis. 
magnetograms indicates that the vertical field frequency attenuation for models $M 1, M 2$ and $M 7$ does not exist. If indeed wavelengths of irregular variations are as high as $6000 \mathrm{~km}$, the conductivity cannot reach values of 1 to $2 \times 10^{-13} \mathrm{em} \mathrm{u}$ at depths of 90 to $200 \mathrm{~km}$.

Because of the difficulties in considering plane-earth induction problems and comparatively local field distributions, plane-earth models of normal induction are of interest. For this purpose models typical of the results obtained by spherical harmonic analysis of the regular magnetic variations have been examined. Figure 7 shows the theoretical power density ratio for a theoretical region consisting of $1 \mathrm{~km}$, conductivity $10^{-11}$ or $10^{-12} \mathrm{emu}$, underlain by material of conductivity $10^{-15} \mathrm{e} \mathrm{m} \mathrm{u}$. At a depth of 200 or $500 \mathrm{~km}$ the conductivity is considered to $10^{-10}$ or $10^{-11}$ e $\mathrm{mu}$. Again the legend is self-explanatory. For a magnetic variation $\Delta Z=\Delta Z_{e}\left(1-\frac{n+1}{n} \cdot \frac{i_{n}}{e_{n}}\right), \Delta X=\Delta X_{e}\left(1+\frac{i_{n}}{e_{n}}\right)$ where $i_{n}, e_{n}$ represent the internal (induced) and external (inducing) term in the potential description of the variation in spherical harmonics of degree $n$. For wave numbers greater than $10^{-8} \mathrm{cms}^{-1}$, the equivalent $n$ is greater than 6 and the approximation $Z=Z_{e}(1-\alpha), X=X_{e}(1+\alpha)$ is valid. The curves in Figure 7 show the variation of $[(1-\alpha) /(1+\alpha)]^{2}$ with period for typical cases. It is clear: (1) that $\alpha$ increases with wavelength and for any wavelength becomes approximately constant at long periods. The differences produced by the depth to the conducting

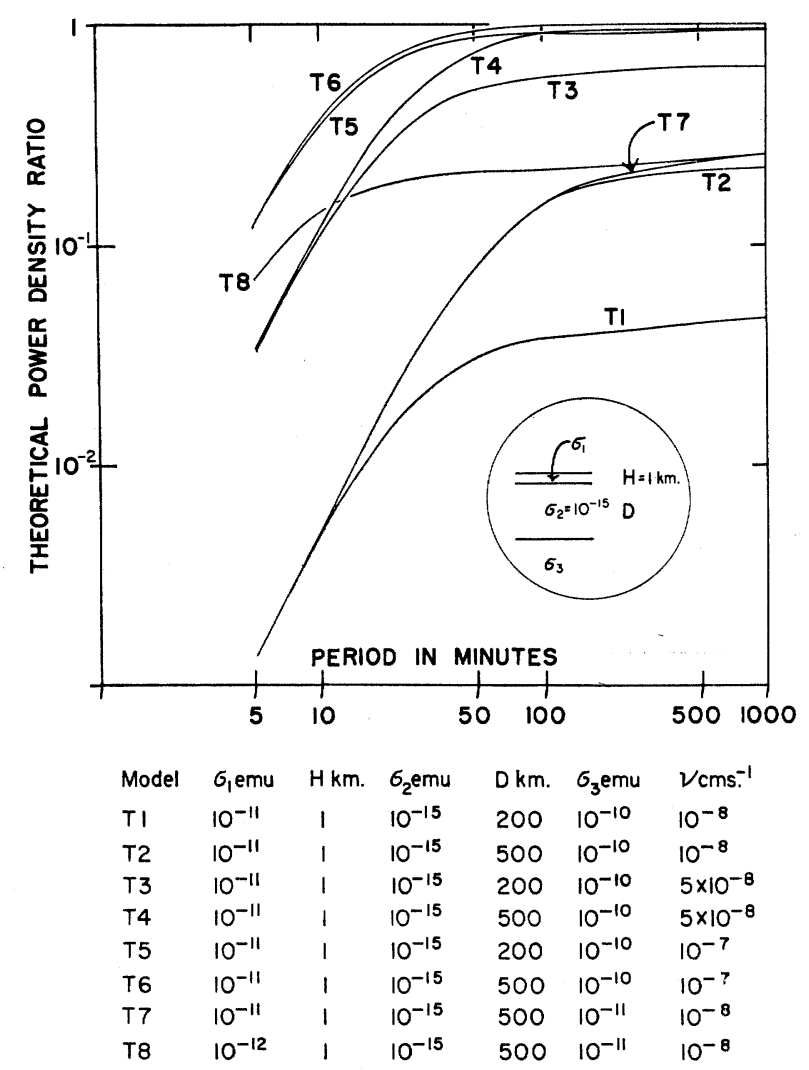

Fig. 7 Calculated power spectral ratios for a non-anomalous region with local field distributions. 
mantle varying are more important at longer periods and at longer wavelengths. (2) that the application of a general correction derived from low order and degree fields (i.e. $\alpha=0.4$ ) can be seriously in error. Values of $\alpha$ from 0.1 to 0.4 have been obtained by geometrical means from chains of stations at auroral latitudes, and are clearly theoretically acceptable with these models. For a long period bay-like disturbance with $\nu \sim 5 \times 10^{-8} \mathrm{~cm}^{-1}, T \sim 100$ minutes, $\alpha=.03$ for a conducting mantle at $500 \mathrm{~km}$ and $\alpha=0.14$ for the same conductor at $200 \mathrm{~km}$ depth. For semi-diurnal periods $\alpha$ varies from 0.4 to 0.6 for these models. (3) that the middle latitude approximation often made that $Z \approx 0$ can be explained at long wavelengths. Note however the effect of surface layer differences between $T 7$ and $T 8$ at periods less than fifty minutes). (4) that the lack of resolution and sensitivity is comparable to that previously noted by other authors. Note, for example, the small differences between models $T 2$ and $T 7$ corresponding to a factor of 10 in the assumed conductivity at $500 \mathrm{~km}$. Models $T 5$ and $T 6$ show that short wavelength irregular variations are incapable of yielding much information about the mantle at a depth exceeding $200 \mathrm{~km}$.

\section{Summary}

A number of models which might account for the Alert anomaly have been studied. The models all require that $\rho \sigma^{1 / 2}$ lie between 5 and $27 \mathrm{em} \mathrm{u}$ were $\sigma$ is the mean conductivity of the anomalous body striking $N E-S W$ and $\rho$ can be interpreted as its physical halfwidth perpendicular to strike.

Simple inductive models, and induction in the mantle driving current along an unusual circuit have been considered. No model quantitatively examined appears capable of accounting for the response asymmetries, noted but as yet poorly defined quantitatively. The circuit hypothesis appears to provide a less reasonable explanation of the magnitude of the inductive term, its frequency response and its phase, than the infinite inductive models, but serious difficulties appear in the latter. In particular the extent of the conductor (now under field examination) is required to be very large, and the temperature required at a comparatively shallow depth appears difficult to reconcile with a stable area.

A comprehensive theory is obviously required considering induction in a body of nonuniform conductivity taking into account its connection to the mantle. This may allow the depth to the top of the body to be increased and overcome the second serious objection mentioned above. Law et al. (1963) have considered magnetotelluric and gravity evidence for the body and briefly discussed the tectonic significance of the anomaly.

The author's earlier explanation of the effect observed on Mould Bay magnetograms has been examined in more detail, and it has been found that the earlier solution $(10-20 \mathrm{~km}$ of material, conductivity $10^{-11} \mathrm{emu}$ at shallow depths) is not substantially changed by taking into account the conductivity of material above and below the body. At the present time a geological explanation (salt beds) cannot be completely eliminated, although the available thickness of sediment appears to be insufficient. A temperature explanation once again leads to difficulties. The assumptions implicit in the approach to date have been listed. Extensive field work is underway at the present time to determine the extent of the effect. 
The application of the same technique to magnetotelluric interpretation and to induction in a non-anomalous region leads to reasonable results.

\section{Acknowlegements}

The writer wishes to acknowledge with pleasure the many useful discussions he has had with Prof. T. Rikitake on magnetic variation anomalies. Professor Rikitake and Dr. E.R. Niblett very kindly criticized this manuscript. Mr. J. DeLaurier contributed very ably to the machine programming involved in this work.

\section{References}

Law, L., DeLaurier, J., Andersen, F. and Whitham, K., 1963. Can. J. Phys. (41, 1868)

Price, A.T., 1962, J. Geophys. Res., 67, 1907

Rikitake, T., 1959 (a), Geophys. J.R. Astr. Soc., 2, 276

1959 (b), Bull. Earthquake Res. Inst., 37, 545

Rikitake, T., 1963 (Private communication)

Schmr.cker, U., 1959, Ab. Akad. d. W.ss. Göttinger, Maths-Phys. Kl. Beit. zum I.G.J., 5, 1

Whitham, K. and Andersen, F., 1962, Geophys. J.R. Astr. Soc., 7, 220

Whitham, K., 1963, Geophys. J.R. Astr. Soc., 3, 26 